\title{
Indices on cryptocurrencies: an evaluation
}

\author{
Konstantin Häusler ${ }^{1}$ D $\cdot$ Hongyu Xia ${ }^{2}$
}

Received: 28 July 2021 / Accepted: 21 January 2022 / Published online: 21 February 2022

(c) The Author(s) 2022

\begin{abstract}
Several cryptocurrency (CC) indices track the dynamics of the rising $\mathrm{CC}$ sector, and soon ETFs will be issued on them. We conduct a qualitative and quantitative evaluation of the currently existing $\mathrm{CC}$ indices. As the $\mathrm{CC}$ sector is not yet consolidated, index issuers face the challenge of tracking the dynamics of a fast-growing sector that is under continuous transformation. We propose several criteria and various measures to compare the indices under review. Major differences between the indices lie in their weighting schemes, their coverage of CCs and the number of constituents, the level of transparency, and thus, their accuracy in mapping the dynamics of the CC sector. Our analysis reveals that simple market cap-weighted indices outperform their competitors. Interestingly, increasing the number of constituents does not automatically lead to a better fit of the $\mathrm{CC}$ sector. All codes are available on Quantlet.com.
\end{abstract}

Keywords Cryptocurrency $\cdot$ Index $\cdot$ Market dynamics $\cdot$ Bitcoin

JEL Classifications C43 · G19

\footnotetext{
Financial support from the Deutsche Forschungsgemeinschaft via the IRTG 1792 "High Dimensional Nonstationary Time Series", Humboldt-Universitaät zu Berlin, is gratefully acknowledged. This research has also received funding from the European Union's Horizon 2020 research and innovation programme "FIN-TECH: A Financial supervision and Technology compliance training programme" under the Grant agreement No. 825215.

Konstantin Häusler

konstantin.haeusler@hu-berlin.de

1 IRTG 1792 "High Dimensional Non-Stationary Time Series”, Humbolt Universität zu Berlin, Berlin, Germany

2 Wang Yanan Institute for Studies in Economics (WISE), Xiamen University, Xiamen, China
} 


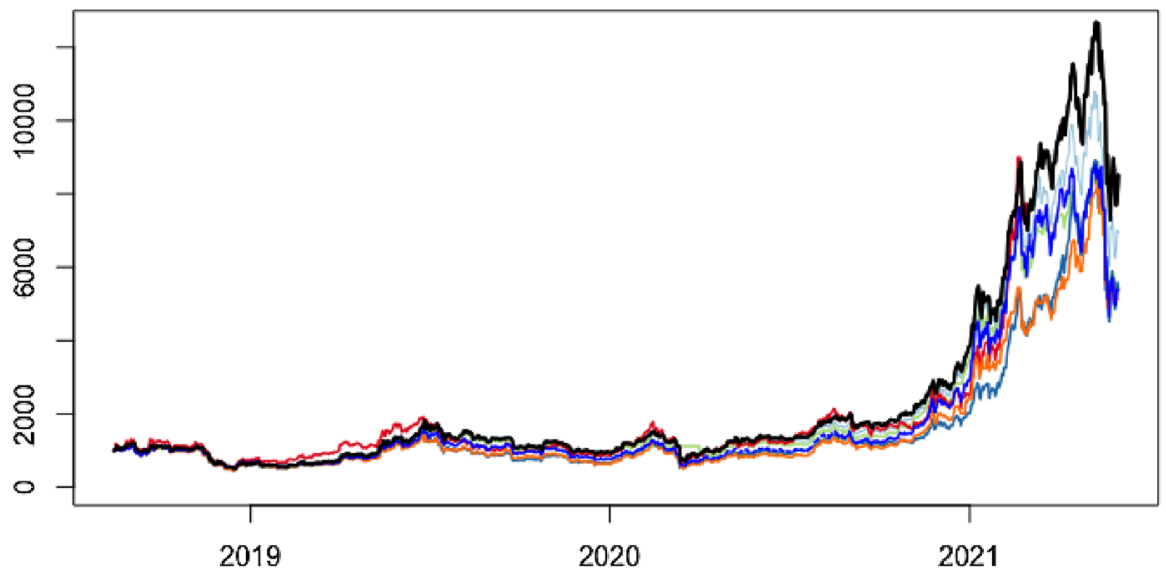

Fig. 1 Scaled CC indices: BBGCI, Bitwise 10, CCi30, CRIX, F5, HODL5, and the total market index (TMI, black solid line). The indices deviate from the TMI due to methodological differences in their construction. $\mathbf{Q}$ evaluation-indices

\section{Introduction}

Indices are widely used to track the market dynamics of financial sectors. Several research groups and companies developed indices for the rising cryptocurrency sector, each of them with different methodological approaches for tracking the highly volatile and dynamic CC market (cf. Härdle and Harvey 2015, p.17; Rivin and Scevola 2018; Elendner 2018). This paper aims to compare and evaluate the characteristics of the currently existing CC Indices: Bloomberg Galaxy Crypto Index (BGCI), Bitwise 10 Large Cap Crypto Index (Bitwise 10), the CRIX, CCi30, F5 crypto index, HODL5, Nasdaq Crypto Index, and Ultra Cap 5 index. Figure 1 displays the index values of the aforementioned indices, scaled to 1000 points at 08/2018.

In this paper, we examine the indices both qualitatively and quantitatively. Starting with the qualitative analysis, we elaborate the methodological differences between the indices. There is no 'right' or 'wrong' in constructing an index; each approach has its raison d'être, but they yield by construction different index values with dissimilar properties. A complete index should contain all available CCs weighted by their market capitalization, though this is practically not feasible because of the high effort involved (trading costs, steady rebalancing, etc.). Therefore, any index issuer must make some simplifying assumptions that affect the properties of the respective index. These methodological differences are elaborated in Sect. 2.3. Ultimately, we group the indices into three groups based on their methodology: purely market cap weighted indices, adjusted market cap weighted indices, and customized weighted indices.

The quantitative analysis follows in Sect. 3. Index values themselves cannot be interpreted meaningfully; therefore, we suggest several comparison criteria: First, accuracy in mapping the dynamics of the $\mathrm{CC}$ sector. We tackle this criteria by constructing a total market index (TMI), which consists of all available 
CCs weighted by market capitalization, and we compute the correlation of each index to the TMI. Additionally, we obtain confidence intervals for the correlations through bootstrapping. Second, we require the indices to have similar statistical characteristics as the TMI. Therefore, we analyze the moments of the return distributions (volatility, kurtosis, and skewness) of each $\mathrm{CC}$ index, which are important indicators for financial decision-makers and investors. Third, based on these moments, we compute several measures, through which we compare the indices quantitatively. The first is the Probabilistic Sharpe Ratio (PSR), introduced by Bailey and Lopez de Prado (2012), which extends the confidence intervals of the well-known Sharpe Ratio by an Edgeworth expansion of higher moments. Thereby, we can assess the robustness and statistical significance of Sharpe Ratios, which is beneficial, because non-normality can inflate the estimates of Sharpe Ratios. In addition, we apply the Sortino Ratio (cf. Sortino and Price 1994) and the Omega Ratio, proposed by Keating and Shadwick (2002), to account in several ways for the non-normality, which is appropriate, since the returns of the CC sector are highly non-normal (cf. Zhang et al. 2018; Petukhina et al. 2021). Another measure we apply is the Maximum Drawdown (MMD), as well an asymmetric risk measure. The MMD is defined as the percentage loss from the local maximum to the local minimum of an index $i$ during a fixed time period. We include this measure, because the $\mathrm{CC}$ sector is not yet consolidated and CCs are nowadays mostly used as speculative investment asset.

Our analysis reveals several insights: First, based on their methodology, we classify the indices in three groups: market cap weighted indices, adjusted market cap indices, and customized weighted indices. Purely market cap-weighted indices perform well. Adjustments in the weighting schemes are not very beneficial. Bitwise 10 and the CRIX, the only purely market cap weighted indices under review, yield very high returns, and their Sharpe Ratios are among the highest of the $\mathrm{CC}$ indices. Introducing a cap/floor to some constituents (cf. BGCI, HODL5) does not only distort the mapping of the CC sector's dynamics; it also affects the statistical properties of indices negatively (i.e., SR, PSR, and MMD). From a methodological point of view, we consider the purely market cap weighting approach as the "correct" one, as it maps the CC sector's dynamics accurately. In practice, this approach is widely employed in classical financial sectors, as indices like the S\&P 500 or the German DAX are constructed in such a way.

Second, a broader coverage of CCs by an index does not automatically yield in a better mapping of the $\mathrm{CC}$ sector's dynamics. The best example is given by HODL5 (5 constituents) and CCi30 (30 constituents): both make adjustments to the market capitalization in their weighting schemes, but HODL5 outperforms CCi30 in terms of accuracy (correlation with TMI). The argument by Rivin and Scevola (2018), an increasing number of constituents yields in more diversification, seems to have been invalidated. This is not surprising, since Keilbar and Zhang (2021) identified several cointegration relationships among the top CCs and Zhang et al. (2018) report correlations among the returns of many $\mathrm{CC}$, especially to Bitcoin. The dynamic adaption through the Akaike Information Criterion (AIC) applied by the CRIX is an effective solution to deal with the ever changing $\mathrm{CC}$ sector, as it balances the index between a small number of constituents and a close adjustment to the entire sector. 


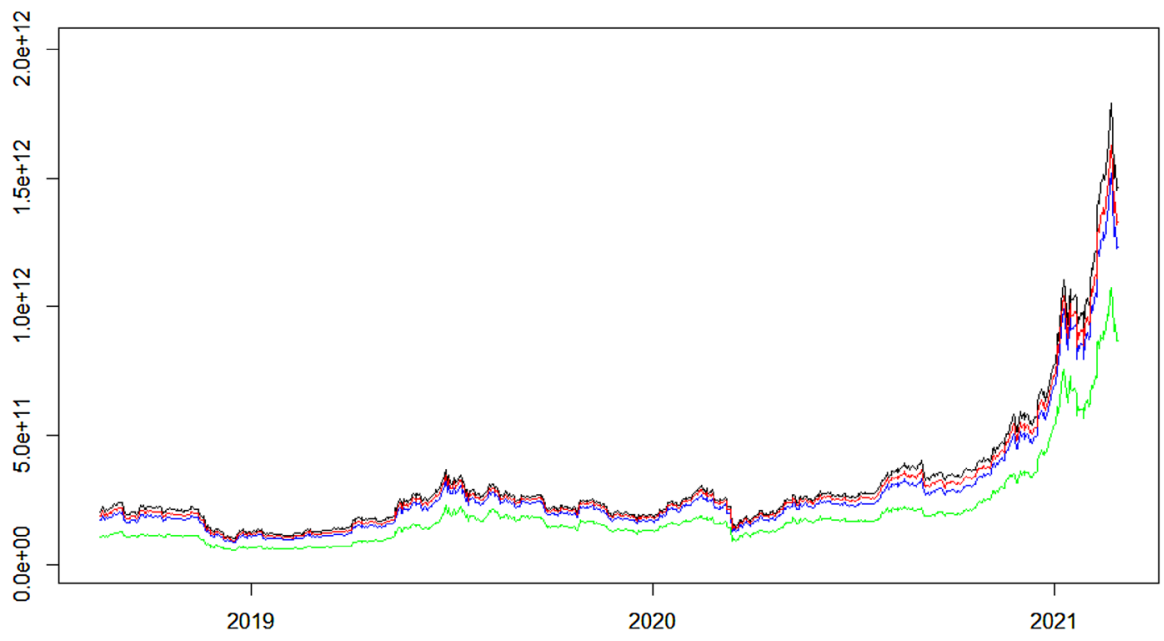

Fig. 2 Market capitalization of the overall CC sector (black line), the top 10 (blue), and top 30 (red) CCs by market capitalization and Bitcoin (green). Data source: coingecko.com. $\mathbf{Q}$ evaluation_indices

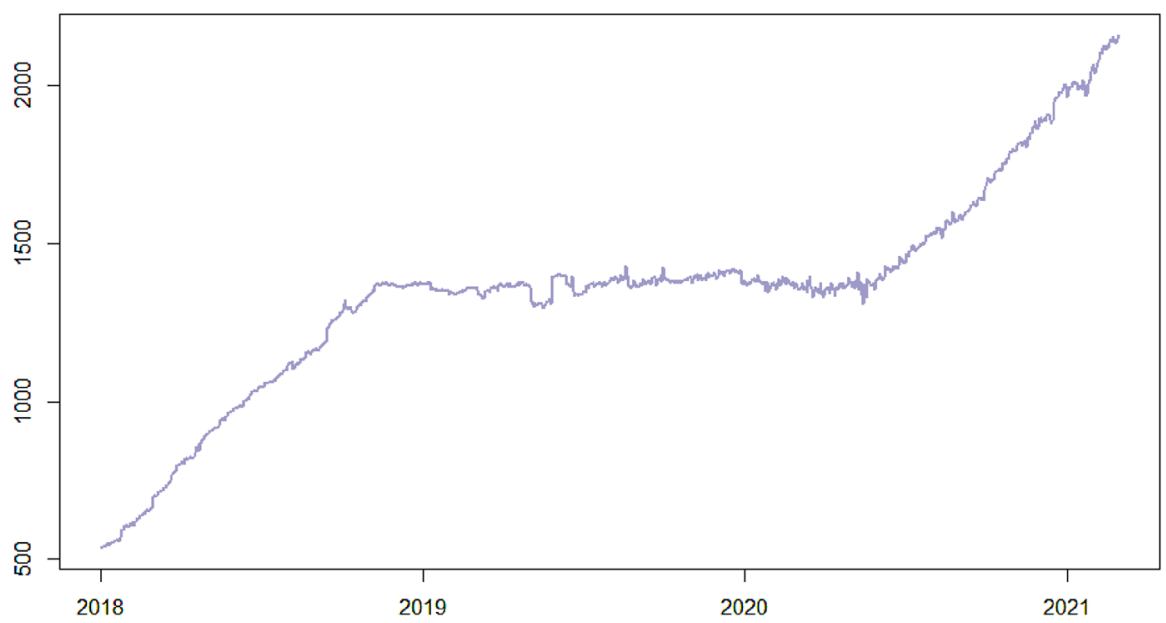

Fig. 3 Number of active coins (= coins with a market capitalization greater than zero) over time. Q evaluation_indices

Third, transparency. The CC sector is not yet consolidated and the underlying technological mechanisms are not understood by the public (Härdle et al. 2020). As for any currency or asset, trust is crucial for the acceptance of CCs. The CC indices could promote trust through transparency, e.g., by disclosing the construction mechanisms, weighting schemes, and index data. Unfortunately, this is not the case for all the indices under review. We do not know which index will emerge as the leading benchmark for the $\mathrm{CC}$ sector, but transparency will certainly be a factor. 
The only index that satisfies all criteria is the CRIX. It is a purely market cap weighted index, its methodological approach is well grounded (cf. Härdle and Trimborn 2015; Trimborn and Härdle 2018), its index data are publicly available (on thecrix.de), it closely tracks the market dynamics (cf. Fig. 5) and by its iterative algorithm, and it optimally benefits from the gains of the rise of the $\mathrm{CC}$ sector (cf. Table 2). As the CC sector is under continuous transition and not yet consolidated (cf. Figs. 3, 4), the CRIX as the only index with dynamic adaption to the CC sector.

The remainder of this paper is structured as follows: Sect. 2 explains the particularities of the $\mathrm{CC}$ sector and introduces the $\mathrm{CC}$ indices under review. Section 3 conducts the quantitative comparison of the indices by several measures. Section 4 discusses the pros and cons of the indices. All codes are available on Q evaluation_indices

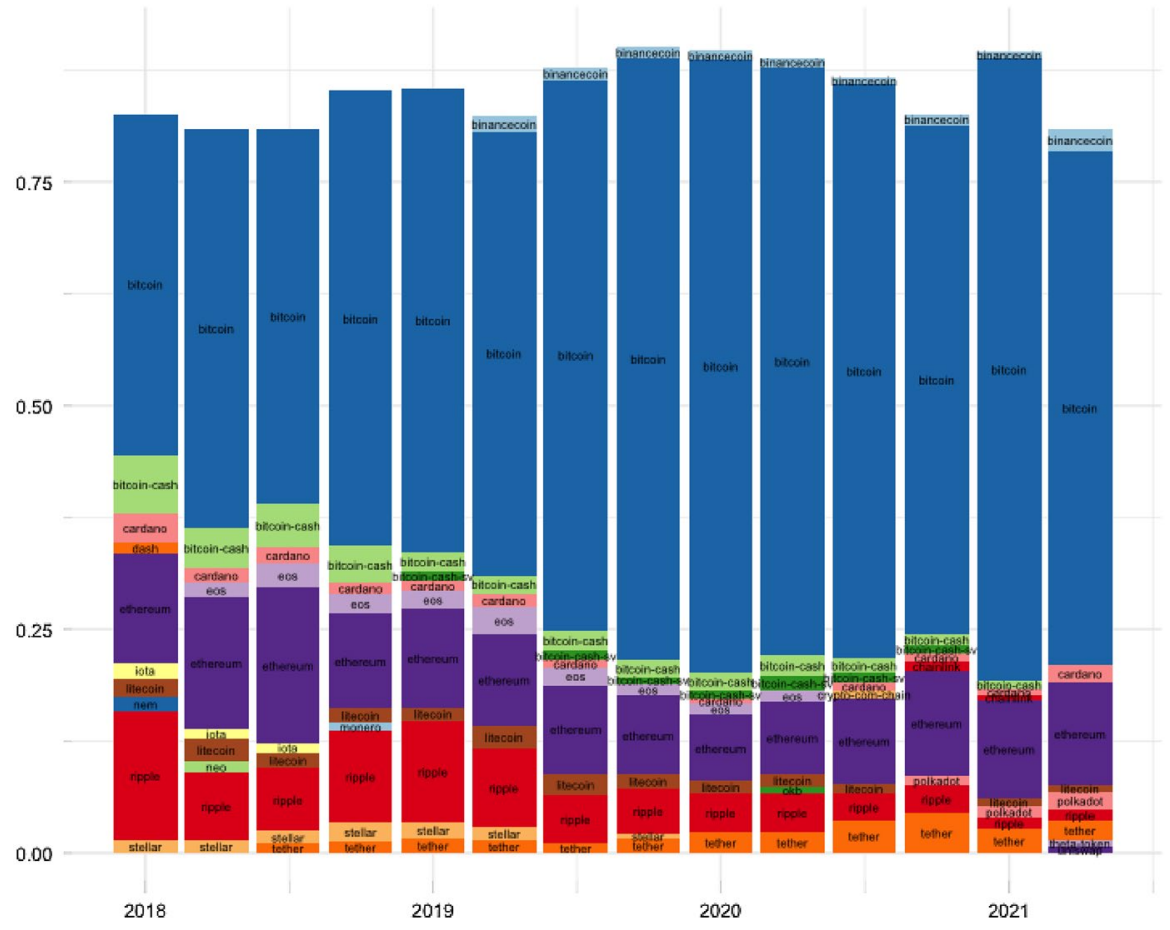

Fig. 4 Share of the top 10 CCs by market cap, over time (quarterly, 2018-01 to 2021-06). Q evaluation_indices 


\section{Data}

\subsection{The CC sector}

As the CC sector is rising, the market capitalization of this sector is steadily growing. By the end of 2020, the overall market cap of the CC sector exceeded \$743 Billion, which is roughly equivalent to Switzerland's gross domestic product. Figure 2 displays the market capitalization of (1) the overall CC sector (black line), (2) the Top 10 CCs (by market cap, blue line), (3) the Top 30 CCs (red line), and 4) Bitcoin (green line). The parallel movement of the time series supports the finding of Keilbar and Zhang (2021), who revealed cointegration relationships among the top CCs. The decoupling of the entire $\mathrm{CC}$ sector from Bitcoin suggests that a new altcoin season could occur in 2021. At least, even though Bitcoin dominates the CC sector (Wang and Vergne 2017; Elendner et al. 2018), it cannot explain the overall market dynamics.

Simultaneously to the growth of the market capitalization, the number of CCs is growing (cf. Fig. 3). The increased attention in 2017/2018 yielded in the evolution of new CCs, and throughout 2019, it seemed that the CC sector consolidated. This phase also includes Paypal's announcement of Bitcoin as a payment method. In mid-2020 though, the creation of new CCs continued, whether this is driven by the economic turmoil caused by the COVID pandemic or the increased acceptance and applications of CCs (Hou et al. 2020; Härdle et al. 2020) remains to be seen.

Besides the growth of the market capitalization and the increasing number of $\mathrm{CCs}$, it is worth noting that the composition of the top 10 and top $30 \mathrm{CCs}$ (by market capitalization) is under constant transformation. Figure 4 displays the composition and share of the top 10 CCs for the period of 2018-01 to 2021-06. Each bar refers to the market share of the top $10 \mathrm{CCs}$ at the first day of each quarter; each colour indicates the share of the respective CC. Some insights are directly observable: the share of Bitcoin changes continuously; in January 2018, Bitcoin represented $38 \%$ of the CC sector, though, in October 2019, its share reflected $67 \%$. Furthermore, the top $10 \mathrm{CCs}$ represent between 80 and $90 \%$ of the overall $\mathrm{CC}$ sector, depending on the time point. But most importantly, only within the 3 years under review, the composition of the top 10 CCs changed very often; only Bitcoin, Bitcoin-Cash, Ethereum, and Ripple were at each stage present in the top 10. In total, $20 \mathrm{CCs}$ appeared in the top 10 in recent years. Mapping these dynamics poses major challenges to index issuers.

\subsection{CC data and index data}

Two data sets are used in the analysis: daily data of all publicly traded CCs are obtained from coingecko.com. The authors thankfully acknowledge their freely accessible API that allows retrieving information on prices, volumes, and market capitalization for each $\mathrm{CC}$. In addition, data on $\mathrm{CC}$ indices are thankfully provided by Bloomberg, f5 crypto capital, $\mathrm{CCi} 30$, and thecrix.de. As the $\mathrm{CC}$ market 
is not centrally organized, no central authority issues an industry benchmark index. The aforementioned index issuers and data providers are partially research units and partially private companies. Their methodological approaches differ significantly, which is the topic of the following paragraphs.

The period of the analysis is restricted to August 2018 to February 2021. The reasoning is rather practically than theoretically founded, because many of the indices have been just recently issued and their historical values are thus not available.

\subsection{Composition of indices}

Bitwise 10 Bitwise 10 Large Cap Crypto Index is a CC index by San Franciscobased company Bitwise Asset Management. Their CC index covers the ten biggest $\mathrm{CCs}$ by market capitalization, not including stablecoins $(=\mathrm{Cs}$ that are pegged to a fiat currency). The index is constructed by weighting each constituent by the free-float and inflation adjusted market capitalization. Unfortunately, the exact weighting scheme is not known to the authors. Bitwise's other indices, Bitwise 20, Bitwise 70, and Bitwise 100, are constructed in a similar manner. Most recently, Bitwise issues an index fund based on Bitwise 10 .

$B G C I$ Bloomberg Galaxy Crypto Index is issued by Bloomberg in cooperation with Galaxy Crypto. The index consists of at most 12 constituents, which are the biggest CCs ranked by market capitalization. The contribution of each constituent to the final index is capped at $40 \%$ and floored by $1 \%$. Unfortunately, Bloomberg does not publish its guidelines on which base they determine the number of constituents of its index. The index values are calculated by

$$
\mathrm{BGCI}_{t}=\frac{\sum_{i=1} P_{i, t} \times Q_{i, m} \times C F_{i, m}}{D},
$$

where $P_{i, t}$ is the price of constituent $i$ at time $t, Q_{i, m}$ the circulating supply of each constituent $i$ in month $m$ and $\mathrm{CF}_{i, m}$ the cap/ floor correction factor; $D$ is a divisor for scaling. If a constituent exceeds the cap, its remaining weight is redistributed among the other constituents relative to their market capitalization.

CRIX The CRyptocurrency IndeX has been developed at the Blockchain Research Center at Humboldt University Berlin by Härdle and Trimborn (2015) and is constructed as a Laspeyres index that weights the market capitalization of its constituents relative to the base year 2015

$$
\mathrm{CRIX}_{t}=\frac{\sum_{i} P_{i t} Q_{i 0}}{\sum_{i} P_{i 0} Q_{i 0}},
$$

where $P_{i t}$ refers to the price of CC $i$ at time $t$ and $Q_{i t}$ to the amount of CC $i$. The number of included coins is adjusted dynamically, to ensure that the CRIX represents the total CC sector accurately. The selection of the constituents is done by an iterative algorithm that penalizes for an increasing number of constituents, but aims to minimize the deviance of the portfolio to the total CC market. As selection criterion, the 
AIC is applied to balance the CRIX between a sparse number of constituents and an accurate representation of the whole CC market. Furthermore, only liquid CCs are eligible to be included on the index.

CCi30 Cryptocurrency Index 30 selects the top $30 \mathrm{CCs}$ by adjusted market capitalization, excluding stablecoins. The index, developed by Rivin and Scevola (2018), is fully transparent and replicable, and historical index values are available on cci30.com.

The weight of each constituent to the index is computed by the square root of its adjusted market capitalization relative to the adjusted market capitalization of the other constituents. The adjusted market capitalization itself is smoothed exponentially to represent a moving average over past periods and to remove temporary fluctuations. Formally, the index values are calculated by

$$
\mathrm{CCi} 30_{t}=\sum_{j=1}^{30} \frac{\sqrt{M_{j}^{*}(t)}}{\sum_{i=1}^{30} \sqrt{M_{i}^{*}(t)}} \frac{P_{j}(t)}{P_{j}(0)},
$$

where $P_{j}$ is the price of constituent $j$ and the adjusted market capitalization $M^{*}$ is weighted in the following way:

$$
M^{*}(t)=\frac{\sum_{i=0}^{\infty} M(T-i) e^{-\alpha i}}{\sum_{i=0}^{\infty} e^{-\alpha i}} .
$$

Rivin and Scevola (2018) argue that the dominance of Bitcoin and Ethereum is reduced by taking the square root of the adjusted market capitalization of each coin. Following their argumentation, smaller CCs beyond Bitcoin and Ethereum get thereby more weight and the index is more diversified.

F5 The F5 crypto index issued by Berlin-based start-up F5 Crypto Capital (cf. Elendner 2018) is a CC index which consists of the 12 biggest CCs by market capitalization. Excluded are stablecoins, anonymous CCs, and CCs that are traded less than 100 days. The weights of each constituent to the F5 index is computed by its momentum

$$
\text { momentum }_{t}=\text { price }_{t}-\text { price }_{t-n} .
$$

The weight allocation by the momentum strategy already reveals the purpose of the index as an investment tool: the best-performing CCs get more weight. Jegadeesh and Titman (1993) have shown that the momentum strategy works for stock markets; however, it still lacks a theoretical foundation. The quantitative analysis in Sect. 3 will elaborate whether the momentum strategy also succeeds in the CC sector.

HODL5 The CC index HODL5 is issued by Amun Technologies Limited, and aims to track the performance of the top 5 most liquid CCs. Interestingly, CCs are not selected according to their current market capitalization, but according to their projected market capitalization in 2050 . The index is constructed using the Laspeyres' form 


$$
\operatorname{HODL5}_{t}=\frac{\sum_{i=1} P_{t, i} * Q_{t, i} * C F_{t, i}}{D_{t}}
$$

where $P_{t, i}$ refers to the price of CC $i, Q_{t, i}$ is amount of CC $i$ outstanding, $\mathrm{CF}_{t, i}$ is weighting cap/floor correction factor, and $D_{t}$ is the stability and scaling divisor. The cap/floor correction factor is imposed to guarantee diversification and avoid underand over-weighting.

NCI The Nasdaq Crypto Index is developed by Nasdaq, Inc. and the crypto asset manager Hashdex and they offer the corresponding Hashdex ETF available on Bermuda Stock Exchange (BSX). Its asset eligibility principles are about core exchanges, core custodians, free-floating trading volume, and price. The index values are calculated by

$$
\mathrm{NCI}_{t}=\frac{\sum_{i}^{C} Q_{t, i} P_{t, i}}{D_{t}}
$$

where $Q_{t, i}$ is the supply of CC $i$ fixed on the previous reconstitution and rebalance date. The divisor $D_{t}$ is used for scaling and smooth transitions at the rebalancing dates. The set of constituents $C$ is not precisely defined. NCI is not included in the quantitative analysis of Sect. 3 , because historical values are not available, since the index was just issued recently.

UC5The CF Cryptocurrency Ultra Cap 5 Index is issued by CF Benchmarks Ltd. It aims to track the performance of the top 5 largest CCs by market capitalization, excluding stablecoins, and aims to be used as a benchmark for the $\mathrm{CC}$ sector as defined by the EU Benchmark Regulations ("EU BMR"). It is calculated as follows:

$$
\mathrm{UC}_{t}=\frac{\sum_{i} a_{i, t} Q_{i, t} P_{i, t}}{D_{i}}
$$

where $a_{i}$ is the free-float adjustment factor, $P_{i, t}$ is the price of constituent $i$ at time $t$, and $D$ is the scaling and smoothing divisor. The Ultra Cap weights its constituents by free float market capitalization. Its historical data are not available.

\subsection{Classification of indices}

A tabular comparison of the index methodologies is summarized in Table 1. The main differences between the approaches concern the number of constituents and their weighting scheme to the respective index. We categorize the CC indices based on their methodological approaches in three categories: First, market cap weighted indices. This group comprises Bitwise 10, the CRIX, NCI, and Ultra Cap 5. Second, adjusted market cap indices. This group comprises BGCI, CCi30, and HODL5. Third, customized weighted indices. This group consists of F5.

The first group, market cap weighted indices, is constructed as Laspeyre indices and constituents are weighted by their market cap. The striking difference between them refers to the selection of constituents: UC5 takes the top 5 CCs by market cap, 
Bitwise 10 the top 10, whereas the CRIX selects dynamically the optimal number of constituents by some AIC/BIC information criteria. The number of constituents of the NCI varies, as well.

The second group, adjusted market cap indices, imposes restrictions on the market cap weighting. This group is as well rather heterogeneous: the number of constituents varies from 5 (HOLD5) to 30 (CCi30). All of them make adjustments to the market caps: the general reasoning is to break the dominance of Bitcoin and Ethereum by limiting their weights (CCi30, BGCI, and HODL5), and thus to allow for more diversification.

The third group, customized weighted indices, consists only of the F5 crypto index. The implementation of the momentum strategy by F5 followed the logic of stock markets (cf. Jegadeesh and Titman 1993; Carhart 1997; Jegadeesh and Titman 2001), and its success (relatively high mean return) indicates that it works as well for the CC sector. The momentum strategy, however, is a purely empirical argument, and it is lacking theoretical foundation.

Some notes on the indices: the methodological approach of the indices is very different. The coverage of CCs varies a lot, from 5 (UC5 and HODL5) to 30 (CCi30). This opens the question whether an increasing number of constituents yield in a better mapping of the overall sector. Furthermore, it remains to be seen whether the adjustments to the market caps (group 2) impact the empirical properties (see Sect. 3), and whether these adjustments are beneficial. On one hand, the adjustments lead to more diversification among the constituents, but at the same time, it may distort the mapping of the $\mathrm{CC}$ sector. Finally, the level of transparency of the indices varies a lot: some index issuers even publish their source code, and others remain mysterious with regard to their mechanisms (choice of constituents, weighting algorithms) and the respective index values. As trust is crucial for financial sectors, we are curious to see which index outperforms its competitors.

\section{Quantitative evaluation of CC indices}

The objective of issuing an index can either be to track a market segment as accurately as possible or to construct an investment instrument on it that allows to diversify coin-specific risks and to benefit from overall market gains. Irrespective of the motivation, several comparison criteria are proposed to measure the performance of the CC Indices under review with regard to both motives.

First, tackling the issue of accuracy, the correlation of each index to the scaled total market index (TMI) is taken as comparison criteria. This benchmark TMI is composed of all available CCs, weighted by their market capitalization, and scaled to 1000 points as starting value. Such a TMI is an ideal theoretical construct; however, it is not feasible to implement it in practice, since there are minimum trading amounts and trading fees.

As can be seen in Fig. 5, all CC indices are highly correlated to the TMI. Each violinplot is obtained by bootstrapping: 1000 samples of 100 observations are taken from the data, for each sample, the correlation between each index and the TMI is 
Table 1 Composition of $\mathrm{CC}$ indices

\begin{tabular}{|c|c|c|c|}
\hline Index & \# of constituents & Index and weights & Abbreviations \\
\hline BGCI & $\leq 12$ & $\mathrm{BGCI}_{t}=\frac{\sum_{i} P_{i, t} \times Q_{i, m} \times C F_{i, m}}{D}$ & $\begin{array}{l}Q_{i, m}=\text { circulating supply of each } \\
\text { constituent } i \text { at month } m \\
C F_{i, m}=\text { Cap/Floor correction factor: } \\
\text { max weight at } 40 \% \text {, min weight } \\
1 \% \text {. }\end{array}$ \\
\hline $\begin{array}{l}\text { Bitwise } \\
10\end{array}$ & 10 & Weighted market cap & \\
\hline $\mathrm{CCi} 30$ & 30 & $\mathrm{CCi} 0_{t}=\sum_{j=1}^{30} \frac{\sqrt{M_{j}^{*}(t)}}{\sum_{i=1}^{30} \sqrt{M_{i}^{*}(t)}} \frac{P_{j}(t)}{P_{j}(0)}$ & $\begin{array}{l}M^{*}=\text { moving-average adjusted } \\
\text { Market Cap of } \mathrm{CC} j\end{array}$ \\
\hline CRIX & $\begin{array}{l}\text { Dynamic adjustment } \\
\text { due to AIC }\end{array}$ & $C R I X_{t}=\frac{\sum_{i} P_{i t} Q_{i 0}}{\sum_{i} P_{i 0} Q_{i 0}}$ & $\begin{array}{l}P_{i t}=\text { price of CC } i \text { at time } t \\
Q_{i t}=\text { supply of CC } i \text { at time } t\end{array}$ \\
\hline F5 & 12 & $\begin{array}{l}\text { Equal weights } \\
+ \text { momentum factor }\end{array}$ & momentum $_{t}=$ price $_{t}-$ price $_{t-n}$ \\
\hline HODL5 & 5 & HODL5 $=\frac{\sum_{i} P_{t, i} Q_{t, i} C F_{t, i}}{D_{t}}$. & $\begin{array}{c}C F_{i}=\mathrm{Cap} / \text { Floor correction factor (if } \\
\text { applicable, otherwise set to } 1 \text { ) }\end{array}$ \\
\hline NCI & Unset & $\mathrm{NCI}_{t}=\frac{\sum_{i} Q_{i, t} P_{t, i}}{D}$ & $\begin{array}{l}Q_{i, t}=\text { supply of asset i fixed on the } \\
\text { previous rebalance date }\end{array}$ \\
\hline UC5 & 5 & $\mathrm{UC5}_{t}=\frac{\sum_{i} a_{i, t} Q_{i, t} P_{i, t}}{D_{i}}$ & $a_{i}$ free-float supply adjustment \\
\hline
\end{tabular}

computed. The highest correlation reveals the CRIX, BGCI, and HODL5. Direct inference from Fig. 5 contains some pitfalls: the TMI itself is constructed as a market cap weighted index (cf. group 1 of Sect. 2.4). The high correlation of TMI with Bitwise 10 and the CRIX are partially caused by the same way of construction.

It is not surprising that all the indices are highly correlated to the TMI; however, it is surprising that HODL5 is higher correlated with the TMI than CCi30. These two indices belong to the second adjusted market cap group. The CCi30 consists of 30 constituents; HODL5 consists only of 5. This finding indicates that it is enough to track the top coins by market capitalization and to discard the rest. Hu et al. (2019) found similar results, and their study showed that the returns of CCs are highly correlated, especially to the returns of Bitcoin. Additionally, Keilbar and Zhang (2021) identified multiple cointegration relationships among the top CCs, which supports the hypothesis that is enough to track a few top coins and to discard the rest.

Second, as the statistical properties of each index differ due to methodological differences of their composition, an analysis of their statistical properties is conducted, with a special focus on their moments. As comparison criteria, the Probabilistic Sharpe Ratio (PSR) introduced by Bailey and Lopez de Prado (2012), the Sortino Ratio (SoR) (cf. Sortino and Price 1994), the Omega Ratio (Keating and Shadwick 2002), and the Maximum Drawdown (MMD) are used as performance measures. A normal Sharpe Ratio as a measure of return to risk is a point estimate constructed on empirical estimates based on historical values. In settings where the moments of the return distribution are non-normal, the classical Sharpe Ratio is not reliable, because higher moments have an impact on the confidence 
intervals of the estimated Sharpe Ratios, and thus on their statistical significance. The proposed measures deal in different ways with the non-normality:

As outlined by Lo (2002), the estimated variance of a Sharpe Ratio under the assumption that returns are normally distributed is given by

$$
\hat{\sigma}(\widehat{\mathrm{SR}})=\sqrt{\frac{1}{n}\left(1+\frac{1}{2} \widehat{\mathrm{SR}}^{2}\right)} .
$$

However, in the overall $\mathrm{CC}$ market, returns are highly non-normal. As shown in Table 2, the properties of all indices differ from the normal distribution. Mertens (2002) suggests to adjust the confidence bands of Sharpe Ratios that are estimated based on non-normally distributed returns by higher moments. Loosening the assumption of normal returns (cf. Bailey and Lopez de Prado 2012), the estimated variance of the Sharpe Ratio extends to

$$
\hat{\sigma}(\widehat{\mathrm{SR}})=\sqrt{\frac{1}{n-1}\left(1-\gamma_{3} \widehat{\mathrm{SR}}+\frac{\gamma_{4}-1}{4} \widehat{\mathrm{SR}}^{2}\right)},
$$

where $\gamma_{3}$ is the skewness and $\gamma_{4}$ the kurtosis. It is basically an Edgeworth expansion that adjusts for the non-normal higher moments. The PSR by Bailey and Lopez de Prado (2012) applies this standard deviation to assess the significance of the estimated Sharpe Ratios. Given a predefined benchmark SR*, the PSR of Bailey and Lopez de Prado (2012) is defined as

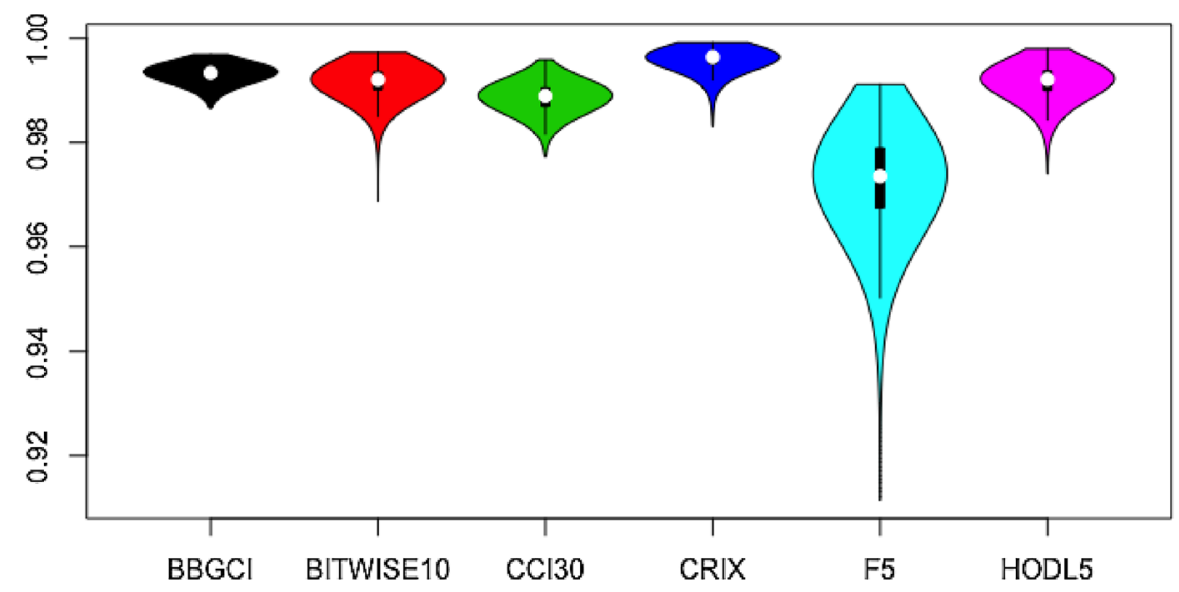

Fig. 5 Violinplots of the correlation between each index and the TMI. Densities obtained through bootstrapping. Q evaluation_indices 


$$
\operatorname{PSR}\left(\mathrm{SR}^{*}\right)=\operatorname{Prob}\left[\mathrm{SR}^{*} \leq \widehat{\mathrm{SR}}\right]
$$

which can be estimated by

$$
\widehat{\mathrm{PSR}}\left(\mathrm{SR}^{*}\right)=Z\left[\frac{\left(\widehat{\mathrm{SR}}-\mathrm{SR}^{*}\right)}{\hat{\sigma}(\widehat{\mathrm{SR}})}\right]=Z\left[\frac{\left(\widehat{\mathrm{SR}}-\mathrm{SR}^{*}\right) \sqrt{n-1}}{\sqrt{1-\gamma_{3} \widehat{\mathrm{SR}}+\frac{\gamma-1}{4} \widehat{\mathrm{SR}}^{2}}}\right],
$$

where $Z$ refers to the cdf of the standard normal distribution.

In addition, Sortino and Price (1994) proposed the Sortino Ratio (SoR) to account for the asymmetric returns of assets. It is a slight modification of the Sharpe Ratio and it measures the riskiness of an asset by the downside deviation $\sigma_{\mathrm{d}}$ instead of the standard deviation

$$
\mathrm{SoR}=\frac{r-r^{*}}{\sigma_{\mathrm{d}}}
$$

The downside deviation $\sigma_{d}$ considers only returns that fall below a certain threshold $r^{*}$

$$
\sigma_{\mathrm{d}}=\sqrt{\frac{1}{N} \sum_{i=1}^{N}\left[\min \left(r-r^{*}, 0\right)\right]^{2}} .
$$

Note, however, that the divisor remains still $N$; thus, the downside deviation measures the size of downside returns, and simultaneously weights them by the probability of their occurrence.

Table 2 Descriptive statistics on daily level for the CC indices under review, rounded values

\begin{tabular}{lllllllllll}
\hline & $\mathrm{G}$ & $r \%$ & $\mathrm{sd}$ & $\gamma_{3}$ & $\gamma_{4}$ & $\mathrm{SR}$ & $\mathrm{PSR}$ & $\mathrm{MMD}$ & $\Omega(0)$ & SoR \\
\hline TMI & & 0.29 & 0.04 & -0.89 & 10.47 & 0.074 & 0.99 & 0.62 & 1.23 & 0.11 \\
BITWISE10 & 1 & 0.23 & 0.03 & -0.85 & 7.56 & 0.066 & 0.98 & 0.59 & 1.22 & 0.09 \\
CRIX & 1 & 0.28 & 0.04 & -0.78 & 10.66 & 0.067 & 0.98 & 0.64 & 1.21 & 0.10 \\
BBGCI & 2 & 0.25 & 0.04 & -0.14 & 5.71 & 0.059 & 0.97 & 0.67 & 1.22 & 0.09 \\
CCI30 & 2 & 0.26 & 0.04 & -0.90 & 9.89 & 0.061 & 0.97 & 0.64 & 1.19 & 0.08 \\
HODL5 & 2 & 0.23 & 0.04 & -0.40 & 5.20 & 0.057 & 0.96 & 0.65 & 1.17 & 0.08 \\
F5 & 3 & 0.32 & 0.05 & -0.66 & 8.20 & 0.071 & 0.99 & 0.65 & 1.22 & 0.10 \\
\hline
\end{tabular}

Period of analysis 08/2018-06/2021

$G$ group (cf. Sect. 2.4), $r$ daily returns, $s d$ standard deviation of returns, $\gamma_{3}$ skewness, $\gamma_{4}$ kurtosis, $S R$ Sharpe Ratio, PSR probabilistic Sharpe Ratio, MMD maximum drawdown, SoR Sortino Ratio. PSR given the benchmark $S R^{*}=0$ (probability of positive Sharpe Ratios). Q evaluation_indices 
A risk to return measure that accounts for non-normal returns but does not require to estimate higher moments individually is the Omega Ratio $(\Omega)$, proposed by Keating and Shadwick (2002). It is defined as the ratio of gains versus losses relative to some threshold $\theta$

$$
\Omega(\theta)=\frac{\int_{\theta}^{\infty}[1-F(r)] \mathrm{d} r}{\int_{-\infty}^{\theta} F(r) \mathrm{d} r},
$$

where $F$ is the empirical distribution function of the returns $r$.

Additionally, we propose the Maximum Drawdown (MMD) as comparison criteria. The MMD measures the peak-to-trough decline of a financial asset $i$ during a fixed period (Magdon-Ismail and Atiya 2004; Ankenbrand and Bieri 2018). As an asymmetric risk measure, it helps portfolio managers to make investment decisions. It is usually defined as the percentage loss of the peak value

$$
\mathrm{MMD}_{i, t}=\frac{M_{i, t}-m_{i, t}}{M_{i, t}},
$$

where $M_{i, t}=\max _{\mu \in[0, t]} P_{i, \mu}$, is the price peak of asset $i$ during time period of $[0, t]$. $m_{i, t}=\min _{\mu \in[0, t]} P_{i, \mu}$, is the price though of an asset during this time period.

Descriptive statistics for all indices whose historical data are available are summarized in Table 2. Unfortunately, we were not able to obtain data of the NCI and UC5. The column returns refers to daily returns, not annualized returns. Column sd displays the standard deviation of daily returns, skewness and kurtosis refer to the higher moments of the return distribution. The Sharpe Ratios (SR) assume a risk-free interest rate $r^{*}=0$. Up to our knowledge, there is still no theoretical founded guideline which interest rate to choose for the computation of Sharpe Ratios in the context of CCs. The Probabilistic Sharpe Ratios PSR compare the Sharpe Ratios relative to the benchmark of $S R^{*}=0$, see Eq. (3). The values in PSR thus refer to the probability of positive Sharpe Ratio estimates. The column MMD is the Maximum Drawdown as defined in Eq. (8). Column $\Omega(0)$ displays the Omega Ratio (cf. Eq. (7)) given a threshold $\theta=0$; thus, it measures the probability of gains versus losses. The last column, SoR, is the Sortino Ratio with a risk-free interest rate $r^{*}=0$.

The estimated returns seem quite low, but one has to consider that these are daily returns. Take TMI as an example, when transforming the daily return to yearly return by compound interest method, the mean yearly return is about $184 \%$. Skewness and kurtosis differ for all indices by far from the normal distribution $\left(\gamma_{3} \neq 0, \gamma_{4} \neq 3\right)$. Our estimates support the findings of Zhang et al. (2018) and Härdle et al. (2020) who report heavy tails of return distributions for many CCs. The non-normality of returns supports the application of the PSR and other asymmetric measures.

Interestingly, the F5 crypto index and CRIX reveal the highest returns, 0.32 and $0.28 \%$, respectively. In this regard, the CRIX comes closest to the TMI, which is not surprising as the iterative construction algorithm of the CRIX aims to minimize the deviance of the returns between the CRIX CC basket and the TMI. 
Furthermore, the high return of the F5 index indicates that the momentum strategy (Jegadeesh and Titman 1993; Elendner 2018) is effective in the CC market.

The moments of some CC indices (CCi30, CRIX) are closer to the TMI than others. This is partially due to the weighting schemes as described in Sect. 2.3 and partially by the coverage of CCs included. Others however (BGCI, HODL5, both Group 2) are closer to the normal distribution.

The highest Sharpe Ratios are achieved by F5 (0.071), the CRIX (0.067), and Bitwise 10 (0.066). Interestingly, this corresponds to Group 1 and Group 3 (cf. Sect. 2.4) . These estimates alone are not very informative, as the return distributions are highly non-normal. The PSR is a useful remedy to work around this issue. Given the benchmark $\mathrm{SR}^{*}=0$, we can interpret the PSR as the probability of obtaining positive Sharpe Ratios. Here, we observe similar patterns: the highest PSR is achieved by F5 (0.99), followed by Bitwise 10 and the CRIX (both 0.98), though the estimated PSRs are quite similar for all indices (due to the overall bullish market dynamics during the period of analysis).

The maximum drawdown, column MMD, measures the maximum percentage peak-to-trough decline of each index and the TMI. The total market's max drawdown is about $62 \%$, and the inglorious highest MMD is achieved by BGCI. CCi30 and the CRIX have the closest MMD value relative to the TMI.

The Omega Ratios for all indices are greater than one, which means that an investment in this sector is likely to yield more profits than losses. For the period of analysis, the $\mathrm{CC}$ sector was bullish, which intuitively confirms our findings. In line with the other measures, the indices of group 1 and 3 reveal the highest Omega values.

So which index performs best? The answer depends on the intentions of the questioner, regulators may prefer most accurate and transparent indices, investors the most profitable ones. That is the topic of the following section, which discusses the (dis-)advantages of the $\mathrm{CC}$ indices.

\section{Discussion of CC indices}

An index that consists of all CCs would perfectly represent the dynamics of the $\mathrm{CC}$ sector. However, this is in practice not feasible due to the high effort involved (steady rebalancing, trading costs, minimum trading amounts, etc.). Therefore, each issuer of an index must make simplifying assumptions; the details are outlined in Sect. 2.3. Consequently, these assumptions affect the statistical properties of the indices, which was the topic of Sect. 3. But which index is now preferable? In this section, we discuss the advantages and disadvantages of the $\mathrm{CC}$ indices, how they approach the challenge of mapping the dynamics of a continuously transforming and rising sector as described in Sect. 2.1, and how accurate, robust, and transparent they are.

Bitwise 10 The index scores with high accuracy in mapping the $\mathrm{CC}$ dynamics and a broad coverage of CCs. From an investor's point of view, the index seems preferable due to its low MMD and high PSR. Interestingly, the plane market cap 
weighting scheme outperformed the adjustments of Group 2. Only a few minor flaws tarnish the picture: the exact weighting scheme is not disclosed and the trading costs can be considerable, as the top $10 \mathrm{CCs}$ by market cap are under constant transformation (cf. Fig. 4)

$B G C I$ The analysis revealed some shortcomings in the construction and properties of the BGCI index. The cap/floor correction factor does reduce the dominance of Bitcoin, though this results in lower (P)SR and high MMD, as the gains of the Bitcoin growth are capped. Overall, BGCI maps the market dynamics very accurately, though it is not quite clear how the number of constituents is determined.

CCi30 Covering the top $30 \mathrm{CCs}$ by market capitalization yields in high diversification and reduces the inherent risks of specific CCs. The disclosure of their methodological approach and the freely accessible index data promote transparency and thereby trust. However, there are two major downsides of this approach: first, the square-root adjustments to the market capitalization of each index distort the mapping of the CC sector dynamics. Second, the extensive list of covered CCs bears relatively high trading costs at each rebalancing date (though trading costs are relatively low in the $\mathrm{CC}$ sector).

CRIX The CRIX is characterized by many positive features: the dynamic adjustment through its iterative algorithm yields in an optimal mapping of the CC dynamics at low costs (i.e., a sparse number of constituents). In addition, this algorithm allows to benefit very closely from the gains of this rising sector, which is empirically supported by the high PSR and the high correlation to the TMI. A major downside lies in the relatively high transaction costs that occur due to the dynamic rebalancing and the in/exclusion of constituents.

F5 The only customized weighted CC index in this analysis performed well. Our prior beliefs that the returns generated by the momentum (cf. Elendner 2018) strategy would be significantly different from the other indices turn out to be validated: the high returns and (P)SR indicate the the momentum strategy works as well for the CC sector. The downside of the momentum strategy, however, is its lack of theoretical foundation. Additionally, the undisclosed customized weighting does not promote transparency.

HODL5 Among the indices with adjustments in their weighting schemes (Group 2), HODL5 reveals high accuracy in mapping the CC dynamics. That is impressive, since the cap/floor correction factor distorts the mapping. However, the index's key figures are rather mediocre.

Overall, the indices deal in different ways with the challenge of mapping the dynamics of the rising $\mathrm{CC}$ sector. Our analysis reveals several insights: First, an increasing number of constituents covered by an index do not automatically yield in a better mapping of the CC sector's dynamics. The best example is given by HODL5 (5 constituents) and CCi30 (30 constituents): both make adjustments to the market capitalization in their weighting schemes, but HODL5 outperforms CCi30 in terms of accuracy. The argument by Rivin and Scevola (2018), an increasing number of constituents lead to more diversification, seems to have been invalidated. This is not surprising, since Keilbar and Zhang (2021) identified several cointegration relationships among the top CCs and Zhang et al. (2018) report correlations among the returns of many $\mathrm{CCs}$, especially Bitcoin. 
Second, purely market cap-weighted indices perform well. Adjustments in the weighting schemes are not very beneficial. Bitwise 10 and the CRIX yield very high returns, and their PSRs are among the highest of the indices under review. Introducing a cap/floor to some constituents like BTC or ETH (cf. BGCI, HODL5) does not only distort the mapping of the $\mathrm{CC}$ sector's dynamics; it also affects the statistical properties of indices negatively (i.e., SR, PSR, and MMD), because capping them also means not benefiting from their strong growth. From a methodological point of view, we consider the purely market cap weighting approach as the "correct" one, as it maps the CC sector's dynamics accurately. In practice, this approach is widely employed in classical financial sectors, as indices like the S\&P 500 or the German DAX are constructed in such a way.

Third, transparency. The CC sector is not yet consolidated and the underlying technological mechanisms are not understood by the public (Härdle et al. 2020). As for any currency, trust is crucial for the acceptance of CCs. The CC indices could promote trust through transparency, e.g., by disclosing the construction mechanisms, weighting schemes, and index data. Unfortunately, this is not the case for all the indices under review. We do not know which index will emerge as the leading benchmark for the $\mathrm{CC}$ sector, but transparency will certainly be a factor.

The only index that satisfies all criteria is the CRIX. It is a purely market cap weighted index, its methodological approach is well grounded (cf. Trimborn and Härdle 2018), its index data are publicly available (on thecrix.de), it closely tracks the market dynamics (cf. Fig. 5) and by its iterative algorithm, and it benefits optimally from the gains of the rise of the CC sector (cf. Table 2). As the CC sector is under continuous transition and not yet consolidated (cf. Figs. 3, 4), the CRIX as the only index with dynamic adaption to the market outperforms its competitors.

\section{Conclusion}

The present paper examined the quantitative and qualitative properties of the currently existing $\mathrm{CC}$ indices. A detailed assessment of their composition, methodological differences, statistical properties, and accuracy in representing the CC sector yielded several insights: First, the major differences in the construction of the indices lie in the weighting scheme and the number of constituents in each index. Surprisingly, a larger number of CCs included in an index do not necessarily lead to higher accuracy in the representation of the CC market. Second, purely market cap weighted indices perform well. Adjustments in the weighting schemes are not very beneficial. Bitwise 10 and the CRIX yield very high returns, and their PSRs are among the highest of the indices under review. Introducing a cap/floor to some constituents does not only distort the mapping of the CC sector's dynamics; it also affects the statistical properties of indices negatively (i.e., SR, PSR, and MMD). We wonder which index will take the dominant position in this sector and act as a benchmark index. In our point of view, the CRIX developed by Trimborn and Härdle (2018) is the most suitable index due to its (1) scientific foundation, (2) transparency and publicly available index data, and (3) its optimal solution to the fundamental 
trade-off every index is facing (a sparse number of constituents and high accuracy in mapping the CC sector's dynamics).

Our analysis can be the basis for further research as well as for practical applications. Our results are of particular interest to financial engineers and financial regulators: the former can build investment tools on CC indices, such as ETFs or options, while the latter can monitor the $\mathrm{CC}$ sector with indices characterized by desirable properties and sound methodological foundations to assess the systemic risk and network effects of the $\mathrm{CC}$ sector. First steps in this direction have been done by Ren et al. (2020) and Guo et al. (2021).

Funding Open Access funding enabled and organized by Projekt DEAL.

Open Access This article is licensed under a Creative Commons Attribution 4.0 International License, which permits use, sharing, adaptation, distribution and reproduction in any medium or format, as long as you give appropriate credit to the original author(s) and the source, provide a link to the Creative Commons licence, and indicate if changes were made. The images or other third party material in this article are included in the article's Creative Commons licence, unless indicated otherwise in a credit line to the material. If material is not included in the article's Creative Commons licence and your intended use is not permitted by statutory regulation or exceeds the permitted use, you will need to obtain permission directly from the copyright holder. To view a copy of this licence, visit http://creativecommons.org/licen ses/by/4.0/.

\section{References}

Ankenbrand, T., \& Bieri, D. (2018). Assessment of cryptocurrencies as an asset class by their characteristics. Investment Management and Financial Innovations, 15(3), 169-181.

Bailey, D. H., \& Lopez de Prado, M. (2012). The Sharpe ratio efficient frontier. Journal of Risk, 15(2), 13.

Carhart, M. M. (1997). On persistence in mutual fund performance. The Journal of Finance, 52(1), 57-82.

Elendner, H. (2018). F5: optimised crypto-currency investment strategies. White paper. Available at https://f5crypto.com

Elendner, H., Trimborn, S., Ong, B., \& Lee, T. M. (2018). The cross-section of crypto-currencies as financial assets: Investing in crypto-currencies beyond bitcoin. Handbook of Blockchain, Digital Finance, and Inclusion (Vol. 1, pp. 145-173). Elsevier.

Guo, L., Härdle, W. K., \& Tao, Y. (2021). A time-varying network for cryptocurrencies. arXiv preprint. arXiv:2108.11921.

Härdle, W. K., \& Trimborn, S. (2015). Crix or evaluating blockchain based currencies. Oberwolfach Reports. https://doi.org/10.4171/OWR/2015/42.

Härdle, W. K., Harvey, C. R., \& Reule, R. C. (2020). Understanding cryptocurrencies. Journal of Financial Econometrics, 18, 181-208.

Hou, A. J., Wang, W., Chen, C. Y., \& Härdle, W. K. (2020). Pricing cryptocurrency options. Journal of Financial Econometrics, 18(2), 250-279.

Hu, A. S., Parlour, C. A., \& Rajan, U. (2019). Cryptocurrencies: Stylized facts on a new investible instrument. Financial Management, 48(4), 1049-1068.

Jegadeesh, N., \& Titman, S. (1993). Returns to buying winners and selling losers: Implications for stock market efficiency. The Journal of Finance, 48(1), 65-91.

Jegadeesh, N., \& Titman, S. (2001). Profitability of momentum strategies: An evaluation of alternative explanations. The Journal of Finance, 56(2), 699-720.

Keating, C., \& Shadwick, W. F. (2002). A universal performance measure. Journal of Performance Measurement, 6(3), 59-84. 
Keilbar, G., \& Zhang, Y. (2021). On cointegration and cryptocurrency dynamics. Digital Finance. https:// doi.org/10.1007/s42521-021-00027-5.

Lo, A. W. (2002). The statistics of Sharpe ratios. Financial Analysts journal, 58(4), 36-52.

Magdon-Ismail, M., \& Atiya, A. F. (2004). Maximum drawdown. Risk Magazine, 17(10), 99-102.

Mertens, E. (2002). Comments on variance of the IID estimator in LO (2002). Technical report, Working Paper University of Basel.

Petukhina, A. A., Reule, R. C., \& Härdle, W. K. (2021). Rise of the machines? Intraday high-frequency trading patterns of cryptocurrencies. The European Journal of Finance, 27(1-2), 8-30.

Ren, R., Althof, M., \& Härdle, W. K. (2020). Tail risk network effects in the cryptocurrency market during the Covid-19 crisis. Available at SSRN 3753421.

Rivin, I., \& Scevola, C. (2018). The CCI30 index. arXiv preprint arXiv:1804.06711.

Sortino, F. A., \& Price, L. N. (1994). Performance measurement in a downside risk framework. The Journal of Investing, 3(3), 59-64.

Trimborn, S., \& Härdle, W. K. (2018). Crix an index for cryptocurrencies. Journal of Empirical Finance, 49, 107-122.

Wang, S., \& Vergne, J.-P. (2017). Buzz factor or innovation potential: What explains cryptocurrencies' returns? PLoS One, 12(1), e0169556.

Zhang, W., Wang, P., Li, X., \& Shen, D. (2018). Some stylized facts of the cryptocurrency market. Applied Economics, 50(55), 5950-5965.

Publisher's Note Springer Nature remains neutral with regard to jurisdictional claims in published maps and institutional affiliations. 